\title{
Harmonic Spline Series Representation of Scaling Functions
}

\author{
Thierry Blu and Michael Unser \\ Biomedical Imaging Group, STI/BIO-E, BM 4.134 \\ Swiss Federal Institute of Technology, Lausanne \\ CH-1015 Lausanne-EPFL, SWITZERLAND
}

\begin{abstract}
We present here an explicit time-domain representation of any compactly supported dyadic scaling function as a sum of harmonic splines. The leading term in the decomposition corresponds to the fractional splines, a recent, continuous-order generalization of the polynomial splines.
\end{abstract}

\section{INTRODUCTION}

The theory of dyadic wavelet decomposition is entirely based on a basic - scaling - function $\varphi(x)$ which is assumed to satisfy good analytic (approximation-wise) properties (partition of unity, stability) together with a geometric condition: a two-scale relation of the form

$$
\varphi(x)=\sum_{k \in \mathbb{Z}} h_{k} \varphi(2 x-k) .
$$

This relation seems to make it almost impossible to express $\varphi(x)$ using standard functions, with the noteworthy exception of the fractional B-spline case which is obtained when $h_{k}=2^{-\alpha}\left(\begin{array}{c}\alpha+1 \\ k\end{array}\right)$ where $\alpha$ is the degree of the spline. ${ }^{1}$ For most standard scaling functions such as Daubechies scaling functions, it is indeed possible to compute the value of $\varphi(x)$ exactly for rational arguments only, but not for irrational values like $\pi$.

In this paper, we show that all compactly supported scaling functions, i.e., most classical scaling functions, can be expressed in an harmonic form, similar to a Fourier series decomposition. As a result it is possible to have an evaluation of a scaling function at any point, not only rational. Moreover, this decomposition uncovers new scaling functions that had never been considered before: the harmonic splines. The result shown here is a development of a similar decomposition that was initially derived by us in another paper ${ }^{2}$

E-mail: thierry.blu@epfl.ch,michael.unser@epfl.ch 


\section{CENTRAL BASIS FUNCTIONS}

In order to derive our harmonic decompostion, we first need to un-localize the scaling function $\varphi(x)$. We will show indeed in this section that any compactly supported scaling function $\varphi(x)$ can be expressed as a digitally filtered version of a self-similar, one-sided but non compactly-supported function $\rho(x)$

$$
\varphi(x)=\sum_{k \geq 0} p_{k} \rho(x-k) .
$$

We will call these "central basis function" by analogy to a similar problem arising in the theory of radial basis functions.

Since $\varphi(x)$ is compactly supported, we will assume with no loss of generality that $h_{k}=0$ for $k \notin[0, L]$. Then, we define the function $\rho(x)$ as

$$
\rho(x)= \begin{cases}\varphi(x) & \text { if } x \in[0,1[ \\ h_{0}^{-j} \rho\left(x 2^{-j}\right) & \text { if } x \in\left[2^{j-1}, 2^{j}[\text { for some } j \in \mathbb{N}\right. \\ 0 & \text { if } x<0\end{cases}
$$

PROPOSITION 1. $\rho(x)$ is self-similar, i.e., it satisfies the property

$$
\rho(x)=h_{0} \rho(2 x)
$$

Moreover, there exists a sequence of coefficients $p_{k}$ such that (2) holds.

Proof. By construction, $\rho(x)$ satisfies the self-similarity property for $x>1 / 2$. Then, because $\varphi(x)=0$ for $x<0$, we simply observe that the scaling relation (1) reduces to $\varphi(x)=h_{0} \varphi(2 x)$ when $0 \leq x \leq 1$ where $\rho(x)$ is identified as $\varphi(x)$. Whence the self-similar relation.

Next, we consider the function

$$
\rho_{0}(x)=\sum_{k \geq 0} r_{k} \varphi(x-k) \quad \text { such that }\left\{\begin{array}{l}
r_{0}=1 \\
r_{n}=h_{0}^{-1} \sum_{k \geq 0} h_{n-2 k} r_{k} \text { for all } n \geq 0 .
\end{array}\right.
$$

Note that $\rho_{0}(x)$ is such that $\rho_{0}(x)=\varphi(x)$ for $x \in[0,1[$. Using the definition of the coefficients $r_{k}$, we easily verify that $\rho_{0}(x)=h_{0} \rho_{0}(2 x)$. As a result, $\rho_{0}(x)=\rho(x)$. Since $\rho_{0}(x)$ is a filtered version of $\varphi(x-k)$, we finally conclude that the reverse is true as well; that is, $\varphi(x)$ is a filtered version of $\rho(x)$.

Conversely, it is a simple matter to verify that $\varphi(x)$ defined by (2) automatically satisfies a scaling relation of the form (1). Whether it is always possible to find a localization filter with coefficients $p_{k}$ such that $\varphi(x)$ is in $\mathbf{L}^{2}$ is still unknown to us; we surmise, though, that the filter defined by

$$
p_{k}=\int_{0}^{\infty} \rho(x) \frac{x^{k}}{k !} e^{-x} d x \quad \text { for } k \geq 0
$$

is a good candidate for this goal. 


\section{HARMONIC DECOMPOSITION}

The self-similar equation (4) is very interesting because, as we observe below, it can be recast into a 1-periodicity condition satisfied by an auxiliary function $u(x)$ :

$$
u(x)=2^{-\alpha x} \rho\left(2^{x}\right) \quad \Longrightarrow \quad u(x+1)=u(x)
$$

where we have let $\alpha=-\log _{2}\left(h_{0}\right)$.

As we know, periodic functions of $\mathbf{L}^{2}$ are equal to their Fourier series decomposition almost everywhere which, in the present case, reads:

$$
u(x)=\sum_{n \in \mathbb{Z}} c_{n} e^{2 i \pi n x} \quad \text { where } c_{n}=\int_{0}^{1} u(\xi) e^{-2 i \pi n \xi} d \xi
$$

We thus obtain the following expression for the central basis function $\rho(x)$ :

$$
\rho(x)=\sum_{n \in \mathbb{Z}} c_{n} x_{+}^{\alpha+\frac{2 i \pi n}{\log _{2}}}
$$

where we have used the definition $x_{+}=\max (x, 0)$. Note that we have an exact expression of $c_{n}$ in terms of $\varphi(x)$, in the case where $\rho(x)$ is obtained from a scaling function $\varphi(x)$ (e.g., Daubechies scaling function):

$$
c_{n}=\frac{1}{\log 2} \int_{1 / 2}^{1} \varphi(x) x^{-\alpha-1-\frac{2 i \pi n}{\log _{2}}} d x .
$$

The harmonic terms $x_{+}^{\alpha+\frac{2 i \pi n}{\log _{2}}}$ can be localized using a generalized finite difference filter. We call "harmonic splines" the functions that are obtained through this process; their Fourier transform takes the following expression:

$$
\hat{\beta}^{\alpha+\frac{2 i \pi n}{\log _{2}}}(\omega)=\left(\frac{1-e^{-i \omega}}{i \omega}\right)^{1+\alpha+\frac{2 i \pi n}{\log _{2}}} .
$$

Notice that these functions are usually not compactly supported. Conversely, we have the identity:

$$
x_{+}^{\alpha+\frac{2 i \pi n}{\log _{2}}}=\sum_{k \geq 0} \frac{\Gamma\left(1+k+\alpha+\frac{2 i \pi n}{\log _{2}}\right)}{k !} \beta^{\alpha+\frac{2 i \pi n}{\log _{2}}}(x-k)
$$

which provides an explicit expression for the coefficients $r_{k}$ of the "un-localization" filter of the harmonic spline. 
Finally, by putting things together, we obtain the main result of this paper:

THEOREM 3.1. Every compactly supported scaling function $\varphi(x)$ can be expressed as a sum of harmonic splines:

$$
\varphi(x)=\sum_{k \geq 0} \sum_{n \in \mathbb{Z}} \gamma_{k, n} \beta^{\alpha+\frac{2 i \pi n}{\log _{2}}}(x-k)
$$

where the coefficients $\gamma_{k, n}$ are defined by

$$
\gamma_{k, n}=c_{n} \sum_{k^{\prime} \geq 0} p_{k-k^{\prime}} \frac{\Gamma\left(1+k^{\prime}+\alpha+\frac{2 i \pi n}{\log _{2}}\right)}{k^{\prime} !}
$$

This result is surprising in a number of aspects:

- A sum of scaling functions is usually not a scaling function; here, it is only because of very particular coefficients that the expression (6) is a scaling function - and that it is compactly supported.

- Usually, (generalized) spline functions appear through a convolution bringing regularity and approximation order to scaling functions ${ }^{3}$; here, they appear through an addition, using quantified complex degrees.

- If we truncate (6) over $n$, we get a function that satisfies the very same two-scale difference equation as $\varphi(x)$; but this approximation is usually not in $\mathbf{L}^{1}$ because its Fourier transform is not continuous at $\omega=0$.

- The expression (6) makes it possible to evaluate standard scaling functions at arbitraryin particular, irrational-values of $x$; as we noticed in introduction, this is unusual for arbitrary scaling functions.

We show in Fig. 1 the behavior of the decomposition formula (6) when we restrict the summation index $n$ to the finite range $[-N, N]$. Note that the fractal structure becomes more apparent and finer grained as one adds more terms to the expansion. Interestingly, the value $\alpha=-\log _{2}\left(h_{0}\right)$ (real part of the degree of the harmonic splines) coincides with the Hölder regularity of Daubechies scaling function.

\section{REFERENCES}

1. M. Unser and T. Blu, "Fractional splines and wavelets," SIAM Review 42, pp. 43-67, January 2000 .

2. T. Blu and M. Unser, "Wavelets, fractals, and radial basis functions," IEEE Trans. Signal Process. 50, pp. 543-553, March 2002.

3. M. Unser and T. Blu, "Wavelet theory demystified," IEEE Trans. Signal Process. 51, pp. 470483, February 2003. 


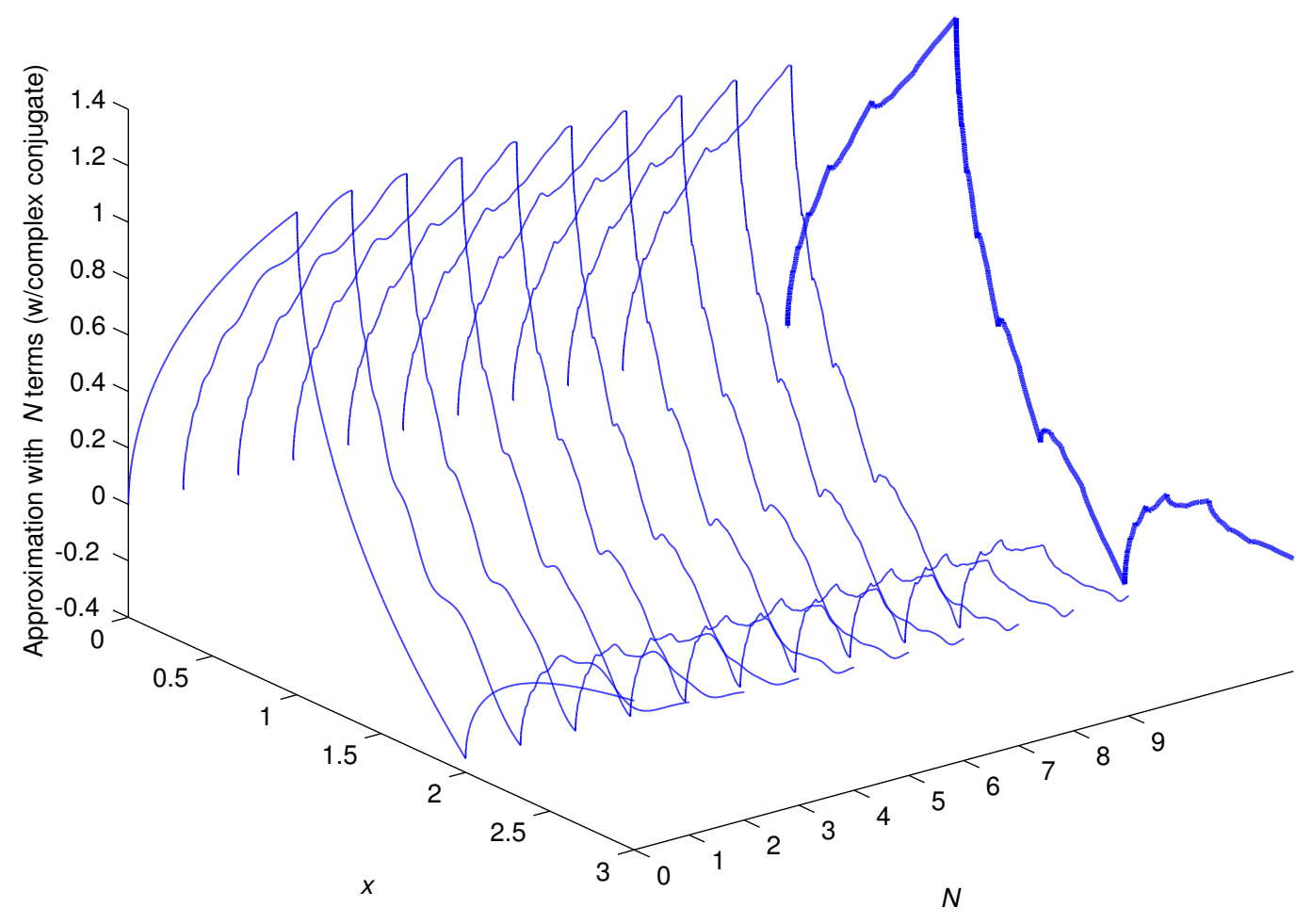

Figure 1. Approximation of Daubechies scaling function of length 4 using the terms $c_{n}$ in (6) for $|n| \leq N$ and for various values of $N=0,1, \ldots 9$. In a bold line, plot of the Daubechies scaling function. 